\title{
PROPOSTA DE MELHORIA BASEADA NA METODOLOGIA DMAIC EM UMA UNIDADE DE PRONTO ATENDIMENTO DE SAÚDE
}

\section{IMPROVEMENT PROPOSAL BASED ON THE DMAIC METHODOLOGY IN A PROMPT HEALTHCARE UNIT}

\author{
L. C. LUIZ ${ }^{1}$, M. DONIN ${ }^{1}$, J. M. L. TYBUSZEUSKY ${ }^{1}$ and D. M. G. CHIROLI ${ }^{1 *}$ \\ ${ }^{1}$ Federal University of Technology Paraná - Ponta Grossa, Department of Production Engineering, Ponta Grossa, \\ Paraná, Brazil
}

${ }^{*}$ Corresponding author. Federal University of Technology, Department of Production Engineering, Ponta Grossa, Paraná, Brazil, Phone: +55 42 3220 7051

e-mail addressl:daianechiroli@utfpr.edu.br(D.M.G.CHIROLI).

\section{A R T I C L E I N F O}

Article history:

Received 2019-10-21

Accepted 2020-01-03

Available online 2020-02-15

palavras-chave

Seis Sigma

Seis Sigma healthcare

Sistema de Saúde Pública

Tempo de atendimento

keywords

Six Sigma

Six Sigma healthcare

Public health system

Time of attendance

\section{A B S T R A C T}

Due to the increasing competitiveness among companies, organizations have new challenges related to the market dispute, and this situation is not only limited to manufacturing organizations, but also covers service organizations. Because to the market dispute, organizations have been increasingly concerned with the quality of service provided and customer satisfaction, seeking to offer customers agility and quality in the delivery of the service. Based on this, the Six Sigma system can assist the organization in reducing variability, increasing customer satisfaction and quality of service provided. In this context, the present work proposes the partial implementation of the DMAIC methodology in a health unit located in Campos Gerais region, in the state of Paraná. Subsequently, the data collection and analysis, process mapping and identification of actions were developed. The calculated sigma level of the process in question was 1,34. Based on this indication, a goal was defined and, consequently, an action plan was developed and presented as a proposal for improvement to the hospital's management. The action plan may suffer some adaptations according to the necessities of the institution.

R E S U M O

Devido à crescente competitividade entre as empresas, as organizações têm novos desafios relacionados à disputa de mercado, sendo que esta situação não se limita apenas a organizações fabris, mas também abrange organizações prestadoras de serviço. Devido à disputa do mercado, as organizações têm se preocupado cada vez mais com a qualidade do serviço prestado e a satisfação do cliente, buscando oferecer aos clientes agilidade e qualidade na entrega do serviço. Com base nisso, o sistema Seis Sigma pode auxiliar a organização na redução da variabilidade, aumento na satisfação do cliente e na qualidade do serviço prestado. Diante deste cenário, o presente trabalho propõe a implementação parcial da metodologia DMAIC em uma unidade de saúde localizada na região dos Campos Gerais, no estado do Paraná. Posteriormente à coleta e análise dos dados, foi calculado o nível sigma do processo, resultando em um valor de 1,34. A partir deste índice, uma meta foi definida e consequentemente um plano de ação desenvolvido e apresentado como uma proposta de melhoria à direção do hospital, podendo ser aceito ou adaptado de acordo com as necessidades identificadas pela organização. 


\section{INTRODUÇ̃̃̃O}

A competitividade entre as organizações fez com que estas buscassem por inovação e novos modelos de gestão com a finalidade de otimizar seus processos produtivos e eliminar desperdícios, sem perder o foco na qualidade dos produtos e serviços prestados. Metodologias relacionadas a melhoria contínua estão sendo incorporadas nos processos das organizações, com o objetivo de melhorar seu desempenho em todos os aspectos, sendo que uma dessas metodologias é o Seis Sigma. O Seis Sigma pode ser entendido como uma estratégia útil à gerência que tem como foco melhorar seu desempenho e lucratividade, utilizando para isso princípios e técnicas de gestão da qualidade, melhorando processos e produtos (PYZDEK; KELLER, 2010; WERKEMA, 2012).

Alinhado a esta metodologia, o presente artigo visa a aplicação da metodologia DMAIC em uma unidade de saúde de pronto atendimento, portanto, trata-se da aplicação de uma metodologia presente no sistema Seis Sigma em um serviço. Os autores Lovelock e Wright (2003) comentam que os serviços são consumidos ao mesmo tempo que são produzidos, isto é, não é possível separar produção e consumo, bem como possuem a característica de intangibilidade, pois não podem ser vistos ou tocados, e variabilidade, visto que dependem do provedor do serviço e do local onde são ofertados.

Devido às características inerentes dos serviços, as prestadoras de serviços só conseguem atender adequadamente os clientes se forem capazes de entender quais são os aspectos que devem ser melhorados para atender as expectativas dos usuários. Desta forma, diz-se que um serviço de qualidade é aquele que consegue levar a plena satisfação ao cliente (SENAC, 1996; CARVALHO; PALADINI, 2013).

Para que seja possível melhorar a qualidade em serviços de saúde, deve-se focar em satisfação, eficiência e resultados clínicos. As organizações de saúde têm buscado atender seus clientes com mais qualidade e eficiência, aumentando o lucro e reduzindo os custos operacionais. Uma das metodologias de melhoria de desempenho que vem sendo utilizada na prestação de serviços relacionados à saúde é o Seis Sigma. Promover boa saúde e bem-estar é um dos 17 objetivos para o desenvolvimento sustentável, compromisso assumido por diferentes países na sede da ONU em 2015, sendo que a metodologia Seis Sigma pode funcionar como aliada para o alcance dessa meta, pois possibilita maior eficiência nos processos de atendimento à saúde, sendo esta a metodologia que norteia a execução do presente trabalho (MALAGÓNLONDONO; MOREIRA; LAVERDE, 2003; TOLGA TANER; SEZEN; ANTONY, 2007; ORGANIZAÇÃO DAS NAÇÕES UNIDAS, 2018).

Através da metodologia DMAIC, presente no sistema Seis Sigma, serão analisadas as operações da unidade de saúde, buscando por oportunidades de melhoria que levem ao aumento da qualidade do serviço. Este trabalho está organizado em 5 tópicos, contando com esta introdução. No tópico 2 tem-se o referencial teórico abordando sobre o seis sigma e o protocolo de Manchester. No tópico 3 a metodologia descreve o caminho traçado para alcançar os objetivos. Os resultados e discussões são apresentados no tópico 4 e, por fim, no tópico 5 as considerações finais acerca do trabalho são discutidas.

\section{REFERENCIAL TEÓRICO}

\subsection{Seis Sigma}

O Seis Sigma é caracterizado por ser um sistema abrangente e flexível para alcançar, sustentar e maximizar o sucesso da organização. Tal condição ocorre quando se compreende as necessidades dos clientes, faz-se uso disciplinado dos fatos, busca-se a melhoria e reinvenção dos processos de negócios (PANDE; NEUMAN; CAVANAGH, 2007).

A metodologia Seis Sigma tem como objetivo que o processo opere em um ambiente livre de defeitos, pois com a eliminação dos defeitos, ocorre a eliminação das insatisfações. A eliminação de defeitos ocorre através da identificação de características que são críticas para a qualidade do processo, pois tais características afetam a percepção do cliente com relação à qualidade do produto ou processo (BRUE, 2005; RAISINGHANI et al., 2005; MARQUES, 2013).

$\mathrm{Na}$ área da saúde, a qualidade do serviço prestado é de grande relevância, e por este motivo o programa Seis Sigma tem se tornado um aliado, pois trata-se de uma ferramenta que permite monitorar e desenhar as atividades. Além disso, o Seis Sigma também se enquadra em uma ferramenta de gestão, auxiliando nas questões de custos com desperdícios e variações existentes nos processos hospitalares (ALENCAR; MACHADO; MARTIN, 2011).

O projeto Seis Sigma Healthcare, pode trazer melhorias a um processo de serviço de saúde como: satisfação do paciente, satisfação do médico, redução de horas extras, redução de espera do paciente, aumento de receita, melhoria da qualidade de vida para os profissionais da saúde, entre outros (TOLGA TANER; SEZEN; ANTONY, 2007).

Os serviços hospitalares são compostos por vários processos, desde o acolhimento do paciente até a efetivação da prestação de serviço, que são realizados por profissionais. Contudo, para que se alcance o nível de excelência, é indispensável a padronização dos processos, incentivando o trabalho em equipe, gerando ganhos em produtividade, ganhos motivacionais e econômicos, bem como a eliminação de desperdícios e do retrabalho (ALENCAR; MACHADO; MARTIN, 2011).

Para que haja a aplicação do Seis Sigma, utiliza-se do método estruturado composto por cinco fases Definir (Define), Medir (Measure), Analisar (Analyze), Melhorar (Improve) e Controlar (Control), dando origem a sigla DMAIC (CONCEIÇÃO et al., 2019; MARTINELLI, 2009; SAKUMOTO, CHIROLI e DZULINSKI, 2019; WHEELER, 2010).

O DMAIC é um método sistemático e disciplinado, que se baseia em dados e no uso de ferramentas estatísticas, sendo que em cada fase do DMAIC são utilizadas diversas ferramentas que ajudam a organização a alcançar os resultados desejados (CARVALHO; PALADINI, 2012; BIAZETTO, CHIROLI, GLAVAM, 2019). 


\subsection{Sistema de triagem de Manchester}

A triagem é uma maneira de gerenciar o risco clinico e direcionar o fluxo de um paciente com segurança (MACKWAY-JONES et al., 2006). O Quadro 1 apresenta o sistema de triagem de Manchester.

Quadro 1 - Protocolo de Manchester

\begin{tabular}{|c|c|}
\hline Classificação & $\begin{array}{c}\text { Tempo de atendimento } \\
\text { máximo }\end{array}$ \\
\hline Emergente & Imediato \\
\hline Muito Urgente & 10 minutos \\
\hline Urgente & 60 minutos \\
\hline Pouco Urgente (ambulatorial) & 120 minutos \\
\hline Não urgente (eletivo) & 240 minutos \\
\hline
\end{tabular}

Fonte: Adaptado de Coutinho, Cecílio e Mota (2012).

O protocolo de Manchester é um sistema de triagem desenvolvido pelo Manchester Triage Group e começou a ser utilizado no Brasil a partir de 2008. Sua classificação possui cinco categorias. Cada categoria recebe um nome, uma cor e um tempo-alvo máximo aceitável até o primeiro atendimento médico (COUTINHO; CECÍLIO; MOTA, 2012).

\section{METODOLOGIA}

O objetivo do presente trabalho é propor melhorias em um ambiente de serviço hospitalar, mantendo o foco em reduzir o tempo de atendimento dos pacientes, utilizando a metodologia DMAIC que está inserida no sistema Seis Sigma.

O ambiente de estudo é um pronto atendimento de um hospital localizado na região dos Campos Gerais, estado do Paraná. O serviço prestado é caracterizado por atendimento Emergencial Pronto Atendimento, com atendimento clínicogeral e ortopedia. O horário de funcionamento do pronto atendimento é de 24 horas por dia, 07 dias por semana e o quadro de funcionários é composto por quatro técnicos de enfermagem, dois enfermeiros, um auxiliar de medicação e dois médicos.

Cada fase do DMAIC demanda a utilização de ferramentas específicas para que seja possível sua aplicação, sendo que a fase "definir" é a primeira a ser implementada e a fase "controlar" é a última a ser implementada. A fase posterior é executada apenas quando a anterior foi concluída, sendo esta a metodologia adotada pelo presente trabalho. Desta maneira, as ferramentas utilizadas na execução de cada fase do presente trabalho, podem ser observadas no Quadro 2.

A fase de definição consistiu no desenvolvimento de uma equipe para que o projeto tivesse andamento conforme o esperado, definindo as metas a serem alcançadas. Para este fim, utilizou-se a ferramenta Project Charter, que reúne as informações mais pertinentes do projeto. Além disso, faz-se necessário observar o funcionamento do processo de atendimento do paciente, desde o momento que chega no pronto atendimento até o momento em que é diagnosticado e liberado. Para tal, utilizou-se a ferramenta SIPOC, possibilitando compreender melhor o fluxo de trabalho, bem como as entradas e saídas do processo.
Quadro 2 - Protocolo de Manchester

\begin{tabular}{|c|c|}
\hline Fases do DMAIC & Ferramentas \\
\hline Definir & Project Charter e SIPOC \\
\hline Medir & $\begin{array}{c}\text { Coleta de dados dos } \\
\text { pacientes }\end{array}$ \\
\hline Analisar & $\begin{array}{c}\text { Estratificação, diagrama } \\
\text { de Pareto, gráfico de } \\
\text { controle, cálculo do nível } \\
\text { sigma, Brainstorming, } \\
5 \mathrm{~W} 1 \mathrm{H}\end{array}$ \\
\hline
\end{tabular}

Fonte: Autoria própria

Após a fase de definição, foram coletados três tipos de horários nas fichas que acompanham o paciente durante seu atendimento, para que fosse possível medir o processo. Os horários coletados nas fichas dos pacientes são referentes à chegada do paciente no pronto atendimento (chegada a recepção), horário que o paciente passou pela triagem e o horário mais tarde que constava na ficha de atendimento.

Os dados foram coletados com base no histórico dos atendimentos realizados nos dez primeiros dias dos meses de abril e junho do ano de 2017, sendo que a equipe do projeto definiu que a quantidade a ser coletada seria de $10 \%$ do total de atendimentos mensais realizados pela unidade de saúde, logo foram coletados 793 atendimentos registrados.

Utilizando os dados coletados nas fichas dos pacientes, estes foram estratificados, pois o atendimento realizado no setor de pronto atendimento do hospital segue uma classificação chamada de protocolo de Manchester, contando com 5 tipos de atendimento: eletivo, ambulatorial, urgência, muita urgência e emergência, sendo que para cada uma dessas classificações estão relacionados tempos máximos para prestação de socorro, de modo que tal classificação possibilite tratar como prioridade e atender de maneira mais rápida os casos mais graves. A estratificação foi realizada com auxílio do software Microsoft Excel.

Foi elaborado na sequência o gráfico de Pareto para compreender melhor a distribuição dos dados coletados, indicando a frequência de ocorrência dos atendimentos ambulatoriais, eletivos, emergenciais, urgentes e de muita urgência. Para execução deste gráfico o software Minitab 17 foi utilizado.

Afim de verificar se os dados coletados eram normais ou não-normais, o software Minitab 17 foi utilizado. Neste mesmo software foram feitos os ajustes necessários nos dados para que algumas ferramentas pudessem ser aplicadas posteriormente, como o gráfico de controle.

Utilizando como base os dados estratificados, calculouse o nível sigma do processo, uma das métricas presentes no sistema Seis Sigma. Realizou-se, então, entrevistas com o corpo técnico do hospital para buscar as causas do problema, prosseguindo para a construção da ferramenta $5 \mathrm{~W} 1 \mathrm{H}$ com o objetivo de encontrar melhorias para o problema levantado.

Após a construção da ferramenta $5 \mathrm{~W} 1 \mathrm{H}$, aquilo que foi levantado pelo trabalho foi levado à direção e administração do hospital, com o objetivo de realizar uma validação daquilo que foi apontado pelo trabalho. Isto ocorreu através de uma reunião com os responsáveis pelo hospital. 


\section{RESULTADOS E DISCUSSÕES}

A primeira fase foi a definição, onde determinou-se como objetivo do projeto a redução do tempo de atendimento dos pacientes, sendo esta meta definida após uma conversa realizada com a direção do hospital. A ferramenta project charter foi elaborada com o objetivo de definir a equipe que faria parte do projeto. Uma equipe ideal para o projeto seria composta por um funcionário de cada setor, ou seja, um enfermeiro, um técnico de enfermagem, um médico e um diretor, porém não foi possível envolver todos estes funcionários na equipe responsável. Devido a esta indisponibilidade, somente a direção e uma supervisora de enfermagem estavam cientes que o projeto estava ocorrendo nas dependências do hospital. O Quadro 3, apresenta o project charter do projeto.

\section{Quadro 3 - Project charter}

\begin{tabular}{|c|c|}
\hline Título do Projeto & $\begin{array}{c}\text { Redução do tempo de espera por } \\
\text { atendimento em uma unidade de saúde } \\
\text { de pronto atendimento }\end{array}$ \\
\hline $\begin{array}{c}\text { Motivos da escolha } \\
\text { do projeto }\end{array}$ & $\begin{array}{c}\text { O tempo total do atendimento para } \\
\text { casos urgentes é maior do que o limite } \\
\text { esperado. }\end{array}$ \\
\hline $\begin{array}{c}\text { Objetivo do Projeto } \\
\text { (Meta) }\end{array}$ & $\begin{array}{c}\text { Reduzir o tempo de atendimento dos } \\
\text { casos urgentes para menos de 60 } \\
\text { minutos até o primeiro mês de 2019. }\end{array}$ \\
\hline $\begin{array}{c}\text { Restrição do } \\
\text { projeto }\end{array}$ & $\begin{array}{c}\text { Apenas atendimentos classificados } \\
\text { como urgentes }\end{array}$ \\
\hline $\begin{array}{c}\text { Membros da } \\
\text { Equipe }\end{array}$ & $\begin{array}{c}\text { Alunos de Engenharia de Produção - } \\
\text { UTFPR PG, Professora orientadora, } \\
\text { enfermeira chefe e direção do hospital. }\end{array}$ \\
\hline $\begin{array}{c}\text { Benefícios } \\
\text { esperados ao } \\
\text { paciente }\end{array}$ & $\begin{array}{c}\text { Atendimento em tempo mais adequado, } \\
\text { aprimoramento da qualidade do serviço } \\
\text { prestado. }\end{array}$ \\
\hline
\end{tabular}

\section{Fonte: Autoria Própria}

Como pode ser observado no Quadro 3, o Project charter elaborado reúne as informações pertinentes sobre a execução do projeto, contendo informações fundamentais para sua execução.

Em posse dos dados coletados nas fichas dos pacientes que receberam atendimento entre os meses de abril e junho de 2017, realizou-se a estratificação dos dados, separando os dados coletados de acordo com o protocolo de Manchester. O protocolo de Manchester aborda que, para atendimentos classificados como urgentes, o máximo que o indivíduo deve esperar para receber atendimento é 60 minutos. Tendo como base este tempo e os dados coletados nas fichas dos pacientes, a meta descrita no Project Charter pode ser especificada.

Para se ter uma visão ampla do fluxo do atendimento da unidade de saúde, utilizou-se a ferramenta SIPOC, descrevendo o processo de atendimento e as partes envolvidas neste processo, conforme mostra a Figura 1.

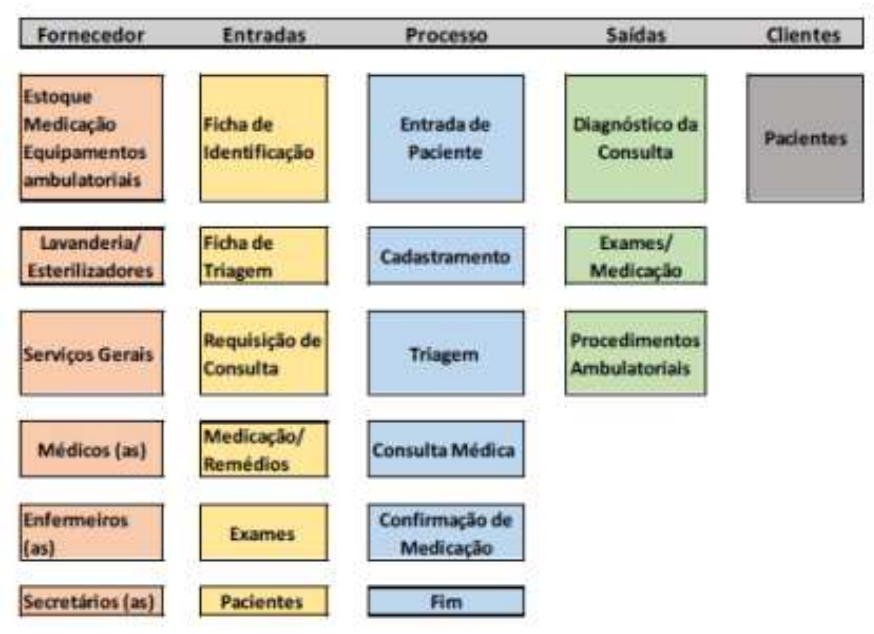

Figura 1 - Ferramenta SIPOC

Fonte: Autoria própria

Como pode ser visualizado na Figura 1, o processo de atendimento é mostrado no centro do SIPOC e esta ferramenta também apresenta os fornecedores do processo, as entradas, saídas e os clientes.

Finalizada a fase "definir", iniciou-se a fase "medir", onde houve a coleta dos dados dos pacientes. Foram coletados três tipos de horários diferentes presentes na ficha dos pacientes: um horário referente à chegada do paciente no pronto atendimento (chegada a recepção), o horário que o paciente passou pela triagem e um último horário que constava na ficha de atendimento, que foi adotado como o horário de saída do paciente. A quantidade coletada foi de 793 fichas de atendimento. O Quadro 4 apresenta os dados estratificados de acordo com o protocolo de Manchester, mostrando o tempo médio total de atendimento em cada nível de risco e o tempo ideal para os atendimentos.

\section{Quadro 4 - Estratificação dos dados coletados}

\begin{tabular}{|c|c|c|c|}
\hline \multicolumn{4}{|c|}{ Atendimentos registrados } \\
\hline & $\begin{array}{c}\text { Número } \\
\text { Total }\end{array}$ & $\begin{array}{c}\text { Tempo } \\
\text { médio (min) }\end{array}$ & $\begin{array}{c}\text { Tempo Ideal } \\
\text { (min) }\end{array}$ \\
\hline Emergente & 6 & 66 & Imediato \\
\hline Muito Urgente & 1 & 58 & 10 \\
\hline Urgente & 197 & 95 & 60 \\
\hline $\begin{array}{c}\text { Pouco Urgente } \\
\text { (ambulatorial) }\end{array}$ & 572 & 99 & 120 \\
\hline $\begin{array}{c}\text { Não urgente } \\
\text { (eletivo) }\end{array}$ & 17 & 93 & 240 \\
\hline
\end{tabular}

Quadro 4 - Estratificação dos dados coletados

De acordo com os dados coletados, 17 fichas das 793 que foram utilizadas para coleta de dados representaram pacientes classificados como "eletivo", sendo que o tempo médio de atendimento para estes 17 "eletivos" foi de 93 minutos. Com base no Quadro 4, pode-se inferir que o tempo médio dos pacientes classificados como "urgente" é superior a 60 minutos, desrespeitando o protocolo de Manchester. Como pode ser observado no Quadro 4, as classificações de "urgência", "muita urgência" e "emergência" não atendem os 
requisitos de tempo máximo definidos pelo protocolo de Manchester, já as demais classificações se encontram dentro das conformidades.

Com o objetivo de observar a distribuição dos dados, a Figura 2 traz o Diagrama de Pareto, onde é possível verificar que $97 \%$ dos atendimentos ocorrem nas classificações de risco "Ambulatorial" e "Urgência". No entanto a classificação "Ambulatorial" possui um tempo médio de 99 minutos, que é menor que o tempo máximo de atendimento adotado pelo que determina o protocolo de Manchester, que define como tempo máximo 120 minutos.

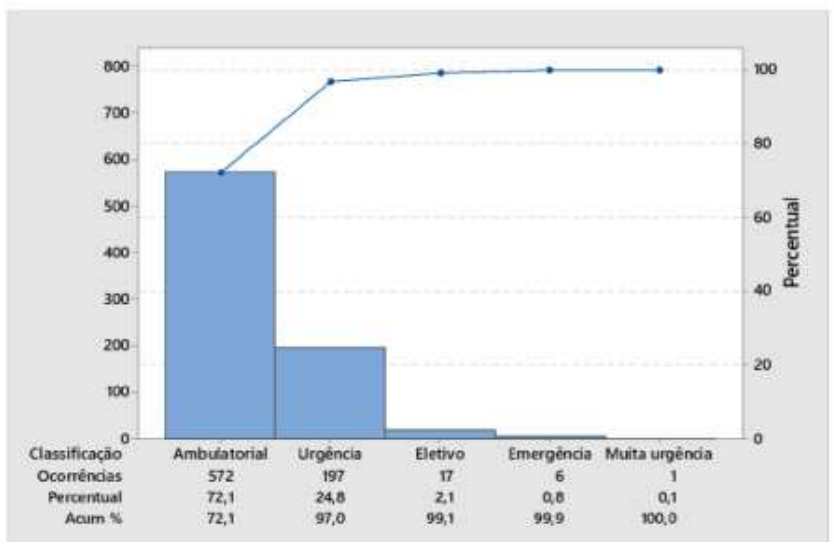

Figura 2 - Diagrama de Pareto dos dados coletados

Apesar de apresentar um número maior de atendimentos na classificação "Ambulatorial”, o pronto atendimento tem como prioridade atender os casos que são urgentes, logo, o trabalho restringiu-se a utilizar as amostras que representam a classificação de risco "urgência", a qual representa quase $25 \%$ do total dos dados coletados. Foi realizada, então, a verificação da normalidade dos dados utilizando o software Minitab 17, bem como foi necessária a utilização da transformação de Box-Cox para elaborar os gráficos de controle para valores individuais e amplitude móvel.

Através da elaboração do gráfico de controle para o processo analisado, foi possível identificar a ocorrência de causas especiais, para que, então, pudesse ser calculado a escala sigma do processo. Devido a identificação de causas especiais que atuaram no processo, foram eliminadas 19 amostras das 197 coletadas para os casos de urgência. Sendo assim, restaram 178 amostras para serem analisadas pelo nível sigma. A compilação do cálculo do nível sigma é apresentada no Quadro 5.

Quadro 5 - Nível Sigma do processo atual

\begin{tabular}{|c|c|}
\hline \multicolumn{2}{|c|}{ Atendimentos Urgentes } \\
\hline TOTAL & 178 \\
\hline Conformes & 78 \\
\hline Não-conformes & 100 \\
\hline DPO & 0,561798 \\
\hline DPMO & 561.798 \\
\hline Nível sigma & 1,34 \\
\hline
\end{tabular}

Uma análise mais profunda dos atendimentos urgentes indica que em 100 casos, de um total de 197, o atendimento foi superior a 60 minutos, representando $55 \%$ da amostra de atendimentos "urgentes". Portanto, como pode ser observado no Quadro 5, 78 atendimentos urgentes foram realizados num período menor ou igual a 60 minutos, sendo considerados como "conformes" e 100 atendimentos foram realizados em um período acima de 60 minutos, sendo considerados como "não-conformes".

Para que seja possível calcular o nível sigma do processo, é necessário calcular a DPO e a DPMO, pois, a partir destes cálculos, a tabela da escala sigma, relacionada ao sistema Seis Sigma, deve ser consultada, para que seja possível determinar o nível sigma do processo. Os cálculos referentes à DPO e DPMO são mostrados nas equações 1 e 2 , respectivamente.

$$
\begin{aligned}
& D P O=\frac{100}{178 \times 1}=0.561798 \\
& D P M O=0,561798 \times 1.000 .000=561.798
\end{aligned}
$$

Para o valor encontrado de DPMO, o nível sigma correspondente é de 1,34, de acordo com a tabela de conversão para a escala sigma. Tais cálculos revelam que, na ocorrência de 1 milhão de atendimentos, 561.798 dos pacientes serão atendidos em um tempo superior a 60 minutos.

Foram então realizadas entrevistas com o corpo técnico do pronto atendimento para que houvesse uma melhor compreensão de quais seriam os motivos que levam à demora no atendimento do paciente na área do pronto atendimento. Foram entrevistados enfermeiros e técnicos de enfermagem de maneira individual.

Por meio das entrevistas pode-se perceber que os funcionários acreditam que a etapa mais demorada, relacionada ao atendimento do paciente, é o atendimento médico. Os funcionários, de modo geral, acreditam que depois da triagem é onde ocorre o maior problema com relação ao tempo de espera por atendimento. Alguns dos entrevistados também apontaram que a demora pode ser decorrente do preenchimento manual de fichas de atendimento e diagnóstico.

Tendo como base as informações levantadas, foi elaborada a ferramenta $5 \mathrm{~W} 1 \mathrm{H}$, para organizar melhor as ideias e conseguir levá-las ao conhecimento dos setores do hospital que são responsáveis pelo pronto atendimento. A ferramenta $5 \mathrm{~W} 1 \mathrm{H}$ pode ser visualizada no Anexo 1, que mostra 4 sugestões para que haja melhoria no processo de atendimento do pronto atendimento estudado.

Após a elaboração da ferramenta $5 \mathrm{~W} 1 \mathrm{H}$, realizou-se uma reunião com os diretores, supervisores e responsáveis pelo hospital, para que as ideias fossem debatidas e concluídas. Nesta reunião foram dadas algumas sugestões para trabalhos futuros e foram feitos alguns questionamentos sobre a coleta de dados do trabalho atual.

\section{CONSIDERAÇÕES FINAIS}

O objetivo principal da realização da pesquisa era identificar alguns pontos que poderiam receber melhorias no processo de atendimento de uma unidade de saúde. A identificação destes pontos foi realizada seguindo a 
metodologia DMAIC, presente no sistema Seis Sigma, e tinha por finalidade reduzir o tempo de espera do paciente por atendimento médico.

Foram realizadas observações do funcionamento do pronto atendimento, entrevistas com os envolvidos no processo, coletados dados referentes ao atendimento dos pacientes, elaboradas ferramentas para realizar a análise dos dados coletados e uma reunião para validar aquilo que havia sido levantado pelo trabalho.

Houveram algumas dificuldades por parte da equipe responsável pelo trabalho na sua execução, como por exemplo a impossibilidade de formar uma equipe multidisciplinar que pudesse orientar o trabalho dentro do hospital, pois apenas poucas pessoas sabiam da ocorrência do trabalho nas dependências do estabelecimento.

Devido a este fato, o trabalho foi baseado quase que inteiramente na visão dos acadêmicos sobre o processo de atendimento do hospital, baseando-se em observações que estes fizeram durante poucas visitas ao pronto atendimento, devido à disponibilidade de tempo para realizar as visitas. $\mathrm{O}$ único momento de validação dos dados ocorreu quando as etapas "definir", "medir" e "analisar" já haviam sido concluídas, o que aconteceu por meio de uma reunião com os administradores do hospital.

Tal etapa de validação dos dados evidenciou falhas na execução do projeto, como a definição de uma métrica não tão confiável, pois os dados coletados não correspondiam à realidade do hospital. Além disso, o processo de atendimento dos pacientes não foi observado o suficiente nem compreendido em sua totalidade, pois uma das sugestões de melhoria levantadas pelo trabalho já havia sido implementada anteriormente pelo hospital. Devido a estes fatores, não foi possível aplicar as etapas "melhorar" e "controlar", como prevê a metodologia DMAIC.

Embora a execução do projeto não tenha atingido o resultados esperado, que era a diminuição do tempo de espera por atendimento médico por parte dos pacientes, como definido no project charter, o trabalho possibilitou realizar o mapeamento do processo, coletar e analisar os tempos de atendimento, identificar potenciais falhas e propor um plano de ação.

Pode-se dizer que o trabalho trouxe aprendizado para a equipe que o executou, no que diz respeito a necessidade de compreender bem o processo no qual se quer realizar alguma melhoria, definir uma métrica confiável que represente a situação real encontrada e realizar validação de dados assim que possível, para que não se chegue ao final do trabalho e percebesse que é necessário refazê-lo por não ter considerado algum fator relevante.

Como sugestão para trabalhos futuros para esta mesma unidade de saúde estudada, pode-se citar a elaboração de alguma métrica que possibilite os gestores tomarem conhecimento da real situação de demora no atendimento, pois não há hoje uma iniciativa neste sentido, sendo que este é um fator importante para que os gestores analisem o desempenho da unidade de saúde e consigam obter um diagnóstico da situação atual encontrada.

\section{R E F E R E N C I A S}

ALENCAR, I. R.; MACHADO, A.D.S.; MARTINS. Programa Seis Sigma: estratégia para melhoria da gestão hospitalar. Disponível em: . Acesso em 13 ago. 2018.

BIAZETTO, F.; CHIROLI, D. M. G; GLAVAM, R.B. Prática da metodologia Seis Sigma para redução de perda na transferência da soja em uma unidade beneficiadora de grãos. Exacta (Uninove), v.17, n. 3, p. 35-60. jul./set. 2019.

BRUE, G. Six Sigma for Managers - 24 Lessons to Understand and Apply Six Sigma Principles in Any Organization, McGraw-Hill, 2005

CARVALHO, M.M.; PALADINI, E.P. Gestão da qualidade: teoria e casos. 2. ed. Rio de Janeiro, RJ: Elsevier; Campus, 2012.

CONCEIÇÃO, R. S.; PARIZ, M. C. SILVA, V. L.; CHIROLI, D. M. G.; ARAGÃO, F. V. Lean Six Sigma: Implementation of improvements to the industrial cost management. Independent Journal of Management \& Production, v.10, n. 6, November - December 2019.

COUTINHO, A.A.P.; CECÍLIO, L.C.O.; MOTA, J.A.C. Classificação de risco em serviços de emergência: uma discussão da literatura sobre o Sistema de Triagem de Manchester, 2012.

LOVELOCK, C.; WRIGHT, L. Serviços: Marketing e Gestão. São Paulo: Saraiva, 2003.

MACKWAY-JONES, K.; MARSDEN, J.; WINDLE, J. Emergency Triage. Manchester Triage Group. $2^{\mathrm{a}}$ ed. Oxford: Blackwell, 2006.

MALAGÓN-LONDONO, G.; MOREIRA, G.M.; LAVERDE, R.G. Administração hospitalar. Rio de Janeiro: Guanabara, 2003.

MARQUES, C.A.N. Monitoramento de Processo Seis Sigma por gráfico de controle de Shewhart. 2013. 80. Dissertação. Universidade Federal de Viçosa, UFV, Minas Gerais.

MARTINELLI, F.B. Gestão da Qualidade Total, Fundação Biblioteca Nacional, 2009. ORGANIZAÇÃO DAS NAÇÕES UNIDAS. Objetivos de desenvolvimento sustentável. Disponível em: < https://nacoesunidas.org/pos2015/ods3/> Acesso 27 set. 2018

PANDE, P. S.; NEUMAN, R. P.; CAVANAGH, R. R. Estratégias Seis Sigma: como a GE, a Motorola e outras grandes empresas estão aguçando seu desempenho. Rio de Janeiro: Qualitymark, 2007.

PYZDEK, T.; KELLER, P. The Six Sigma handbook. - A Complete Guide for Green Belts, Black Belts, and Managers at All Levels. Mc Graw Hill, 2010.

RAISINGHANI, M.S.; ETTE, H.; PIERCE, R.; CANNON, G.; DARIPALY, P. Six Sigma concepts, tools and applications, Industrial Management p Data Systems, 491-505, 2005. SENAC. Departamento Nacional. Qualidade em prestação de serviços, 1996.

SAKUMOTO, S. M.; DE GENARO CHIROLI, D. M.; DZULINSKI, A. C. (2019). Using Lean Six Sigma to Increase Efficiency of a Grain Receipt Process of a Brazilian Agroindustry Cooperative. American Journal of Engineering and Applied Science, Vol. 12 (2): 214.226. DOI:10.3844/ajeassp.2019.214.226.

TOLGA TANER, M.; SEZEN, B.; ANTONY, J. An overview of six sigma applications in healthcare industry. 
International Journal of health care quality assurance, WHEELER, D.J. The Six Sigma Practitioner's Guide to Data v. 20, n. 4, p. 329-340, 2007.

WERKEMA, M.C.C. Criando a cultura Seis Sigma. 2 ed. Belo

Horizonte: Werkema Editora, 2012.

\section{Anexo 1}

PREENCHIMENTO DA FERRAMENTA 5WIH

\begin{tabular}{|c|c|c|c|c|c|c|}
\hline Hipótese & $O$ que fazer? & Por que? & Onde? & Como? & Quem? & Quando? \\
\hline $\begin{array}{l}\text { Mistura de } \\
\text { fichas na } \\
\text { triagem. }\end{array}$ & $\begin{array}{l}\text { Evitar que } \\
\text { fichas de } \\
\text { diferentes } \\
\text { triagens scjam } \\
\text { misturadas. }\end{array}$ & $\begin{array}{l}\text { As fichas } \\
\text { misturadas } \\
\text { podem causar } \\
\text { divergência } \\
\text { dos } \\
\text { atendimentos } \\
\text { prioritários. }\end{array}$ & $\begin{array}{l}\text { No pronto } \\
\text { atendimento. }\end{array}$ & $\begin{array}{l}\text { Con feccito de } \\
\text { um espaco } \\
\text { com as } \\
\text { respectivas } \\
\text { cores das } \\
\text { triagens para } \\
\text { melhor } \\
\text { organizaçăo. }\end{array}$ & $\begin{array}{l}\text { Equipe } \\
\text { do } \\
\text { projeto, } \\
\text { direçào } \\
\text { do } \\
\text { hospital, } \\
\text { equipe } \\
\text { técnica. }\end{array}$ & $\begin{array}{l}\text { A partir } \\
\text { de Agos to } \\
\text { de } 2018 \text {. }\end{array}$ \\
\hline $\begin{array}{l}\text { Os médicos } \\
\text { nito conseguem } \\
\text { atender a alta } \\
\text { demanda. }\end{array}$ & $\begin{array}{l}\text { Fazer com } \\
\text { que o } \\
\text { atendimento } \\
\text { médico seja } \\
\text { agilizado. }\end{array}$ & $\begin{array}{l}\text { Os pacientes } \\
\text { esperarầ } \\
\text { menostempo } \\
\text { por } \\
\text { atendimento } \\
\text { médico, } \\
\text { agilizando o } \\
\text { serviço. }\end{array}$ & $\begin{array}{l}\text { No pronto } \\
\text { atendimento. }\end{array}$ & $\begin{array}{c}\text { Separar os do is } \\
\text { medicos que } \\
\text { atendem o } \\
\text { pronto } \\
\text { atendimento, } \\
\text { fizendo com } \\
\text { que um deles } \\
\text { atenda os casos } \\
\text { urgentes e } \\
\text { outro atenda os } \\
\text { casos } \\
\text { ambulatoriais }\end{array}$ & $\begin{array}{l}\text { Equipe } \\
\text { do } \\
\text { projeto, } \\
\text { direçâo } \\
\text { do } \\
\text { hospital, } \\
\text { equipe } \\
\text { técnica. }\end{array}$ & $\begin{array}{c}\text { A partir } \\
\text { de Agosto } \\
\text { de } 2018 \text {. }\end{array}$ \\
\hline $\begin{array}{c}\text { O } \\
\text { preenchimento } \\
\text { mamual das } \\
\text { fichas deixa o } \\
\text { processo mais } \\
\text { lento. }\end{array}$ & $\begin{array}{l}\text { Implementar } \\
\text { integralmente } \\
\text { no pronto } \\
\text { atendimento } \\
\text { software de } \\
\text { sistema de } \\
\text { gestão } \\
\text { presente no } \\
\text { bospital, } \\
\text { soffware de } \\
\text { gestäo de } \\
\text { saúde } \\
\text { presente no } \\
\text { bospital. }\end{array}$ & $\begin{array}{l}\text { Elimina a } \\
\text { necessidade de } \\
\text { preenchimento } \\
\text { manual das } \\
\text { fichas dos } \\
\text { pacientes. }\end{array}$ & $\begin{array}{l}\text { No pronto } \\
\text { atendimento. }\end{array}$ & $\begin{array}{c}\text { Disponibilizar } \\
\text { os recursos } \\
\text { disponiveis no } \\
\text { hospital para } \\
\text { implementar o } \\
\text { software no } \\
\text { pronto } \\
\text { atendimento, } \\
\text { além de } \\
\text { realizar } \\
\text { treinamento } \\
\text { com } \\
\text { enfermeiros e } \\
\text { médicos que } \\
\text { utilizario o } \\
\text { sistema. }\end{array}$ & $\begin{array}{l}\text { Direçào } \\
\text { do } \\
\text { hospitale } \\
\text { equipe } \\
\text { técnica. }\end{array}$ & $\begin{array}{l}\text { A partir } \\
\text { de Agos to } \\
\text { de } 2018 \text {. }\end{array}$ \\
\hline $\begin{array}{l}\text { Fichas sem } \\
\text { assi natura do } \\
\text { módico. }\end{array}$ & $\begin{array}{l}\text { Evitar que as } \\
\text { fichas } \\
\text { cheiguem ao } \\
\text { faturamento } \\
\text { sema } \\
\text { assinatura do } \\
\text { médico. }\end{array}$ & $\begin{array}{l}\text { As fichas que } \\
\text { chegam ao } \\
\text { fituramento } \\
\text { sem assinatura } \\
\text { geram } \\
\text { retraballo para } \\
\text { o faturamento. }\end{array}$ & $\begin{array}{l}\text { No pronto } \\
\text { atendimento. }\end{array}$ & $\begin{array}{c}\text { Elaboração de } \\
\text { uma instruçâo } \\
\text { de trabalho e } \\
\text { colocaçầ em } \\
\text { um local } \\
\text { visivel, para } \\
\text { que os médicos } \\
\text { sempre sejam } \\
\text { lembrados. }\end{array}$ & $\begin{array}{l}\text { Equipe } \\
\text { do } \\
\text { projeto, } \\
\text { direção } \\
\text { do } \\
\text { hospital. } \\
\text { equipe } \\
\text { técnica. }\end{array}$ & $\begin{array}{l}\text { A partir } \\
\text { de agosto } \\
\text { de } 2018 .\end{array}$ \\
\hline
\end{tabular}

